\title{
Variabilidad temporal de la abundancia de Coralliophila abbreviata y Coralliophila caribaea (Mollusca, Coralliophilidae) en un arrecife coralino del Parque Nacional Morrocoy, Venezuela
}

\author{
Carlos del Mónaco ${ }^{1}$, Estrella Villamizar ${ }^{1} \&$ Samuel Narciso $^{2}$ \\ 1. Laboratorio de Estudios Marino Costeros, Universidad Central de Venezuela. Caracas, Venezuela Apdo. Postal 1080; \\ carlosdelmonaco@gmail.com, estrellavillamizar@yahoo.com. \\ 2. Fundación por la Defensa de la Naturaleza. Estado Falcón, Venezuela; snarciso@fudena.org.ve
}

Recibido 20-X-2006. Corregido 07-IX-2007. Aceptado 31-X-2007.

\begin{abstract}
Temporary abundance variation of Coralliophila abbreviata and Coralliophila caribaea (Mollusca, Coralliophilidae) in a coral reef at Morrocoy National Park. The mollusks Coralliophila abbreviata and $C$. caribaea are coral predators in the Caribbean Sea . We evaluated the temporal variability of abundance and size structure of C. abbreviata and C. caribaea in Cayo Sombrero, Morrocoy National Park, Venezuelar (September 2004 - August 2005). The density of C. abbreviata and C. caribaea was evaluated monthly by means of two 50- meter long transects paralell to the coast line, at $3 \mathrm{~m}$ and $6 \mathrm{~m}$ deep; aditionally, 30 individuals of C. abbreviata were collected to measure size. The densities of C. abbreviata at the shallow transect fluctuated between $1.78 \mathrm{ind} / \mathrm{m} 2$ in September and $6.26 \mathrm{ind} / \mathrm{m} 2$ in May. The densities at the deep transect fluctuated between $4.23 \mathrm{ind} / \mathrm{m} 2$ in January and $7.66 \mathrm{ind} / \mathrm{m} 2$ in May. The densities of C. caribaea at the shallow transect fluctuated between $0 \mathrm{Ind} / \mathrm{m} 2$ (except in September) and $0.03 \mathrm{Ind} / \mathrm{m} 2$ (September) and the densities at the deep transect fluctuated between $0 \mathrm{Ind} / \mathrm{m} 2$ (October to March and July) and $0.1 \mathrm{Ind} / \mathrm{m} 2$ (April). Most individuals of C. abbreviata and C. caribaea measured between 12 and $16 \mathrm{~mm}$ and between 10 and $15 \mathrm{~mm}$ long respectively. Rev. Biol. Trop. 56 (2): 501-511. Epub 2008 June 30.
\end{abstract}

Key words: Coralliophila sp, corallivorous, temporal variability, abundance, size structure, Morrocoy National Park.

Las poblaciones de muchos invertebrados marinos presentan frecuentemente fluctuaciones en sus abundancias poblacionales. Dichas fluctuaciones pueden surgir a partir del diferencial entre la supervivencia y la mortalidad en cada uno de los estadios del ciclo de vida y puede ser atribuible a los procesos que afectan el período reproductivo y/o estadios tempranos de sus ciclos de vida (Turner 1994b). Específicamente, el aporte de larvas (tasas de desarrollo larval, dispersión hidrodinámica y concentración de larvas), procesos de establecimiento (competencia por establecimiento, preferencias de sustrato, tasas de reclutamiento) y mortalidad post reclutamiento (Turner 1994b). La variabilidad en el número de larvas sobrevivientes luego de su establecimiento puede ser un factor determinante en la definición de las fluctuaciones del tamaño de una población de adultos de muchos invertebrados marinos con estadios planctónicos (Turner 1994a).

Las especies del género Coralliophila son moluscos coralívoros comunes en los arrecifes coralinos del Caribe. Se encuentran en aguas someras y habitualmente permanecen en agregaciones por encima de veinte individuos sobre las colonias coralinas vivas (Ward 1965), en la frontera entre el tejido vivo y el tejido muerto erosionado (Lewis 1960). Estos moluscos se alimentan de tejido coralino mediante una probóscide que insertan sobre la cavidad celentérica del coral que, a través de una acción enzimática, provoca la fragmentación del epitelio. Se alimentan de por lo menos 14 especies 
de corales escleractínidos (Miller 1981), entre los cuales sus presas principales son Acropora palmata y Acropora cervicornis que son dos especies de amplia importancia ecológica en el Caribe por su gran abundancia y por ser formadoras de arrecife (Miller 2001). Se ha estimado que C. abbreviata puede consumir $9 \mathrm{~cm}^{2} \mathrm{de}$ tejido coralino vivo en 24 horas (Ott \& Lewis 1972) y su abundancia y características poblacionales varían en función de la abundancia y presencia de la especie-presa del coral y además de la localidad geográfica (Hayes 1990).

El objetivo del presente estudio fue evaluar la variabilidad temporal de la abundancia y los cambios en la estructura de talla a lo largo del año de C. abbreviata y C. caribaea en la localidad de Cayo Sombrero del Parque Nacional Morrocoy, Venezuela.

\section{MATERIALES Y MÉTODOS}

El Parque Nacional Morrocoy se encuentra ubicado en la costa noroeste (NW) de Venezuela $\left(10^{\circ} 52^{\prime} \mathrm{N}, 69^{\circ} 16^{\prime} \mathrm{W}\right)$. Este parque abarca una superficie total de $320 \mathrm{~km}^{2}$ de ecosistemas continentales, insulares y marinos entre los que destacan bosques de manglar, dominados por Rhizophora mangle, praderas de pastos marinos dominadas por Thalassia testudinum, fondos arenosos desprovistos de vegetación y arrecifes coralinos costaneros. El clima es relativamente constante durante todo el año. La temperatura del aire está acotada entre 20 y $30^{\circ} \mathrm{C}$, mientras que la precipitación presenta un régimen bimodal con mínimos que varían entre 8 y $20 \mathrm{~mm}$ durante enero-marzo, y máximos que oscilan entre 60 y $65 \mathrm{~mm}$ durante septiembre-noviembre. La humedad relativa es alta (83-90\%) con los máximos valores registrados entre mayo y agosto, y la velocidad del viento varía entre 1.5 y $2.5 \mathrm{~ms}$, con dirección noreste-suroeste durante casi todo el año. La temperatura del agua varía entre 26 y $29^{\circ} \mathrm{C}$ en la superficie y entre 18 y $20^{\circ} \mathrm{C}$ a $150 \mathrm{~m}$ de profundidad. La salinidad oscila entre $36 \mathrm{y}$ $38 \%$, debido a las elevadas tasas de evaporación del área (Bone et al. 1998).

Se colocaron transectas fijas de $50 \mathrm{~m}$ de longitud a profundidades de tres y seis metros cada una (isobatas), y se evaluaron las abundancias de los depredadores mediante cuadrículas de $1 \mathrm{~m}^{2}$, las cuales estuvieron distribuidas dentro de cada isobata de un modo intermitente. Se evaluó cada 10 metros para un total de 30 metros evaluados por transecta. En cada cuadrícula se evaluó exhaustivamente el número de individuos de C. abbreviata y C. caribaea mediante observaciones y tacto sin recolectarlos. Dicha actividad se realizó al inicio de cada mes durante un año.

Se midió el largo y ancho de entre 19 y 27 individuos de C. abbreviata in situ con un vernier en cada mes sin ser recolectados y 53 individuos de $C$. caribaea durante abril sobre una colonia de $A$. palmata (no se midieron mensualmente debido a su muy baja abundancia en el área de estudio). El largo fue medido desde el ápice hasta el canal sifonal y el ancho fue medido sobre la abertura opercular de los organismos; todos los individuos medidos de C. abbreviata estuvieron asociados con la especie coralina Montastraea annularis para evitar el efecto nutricional sobre el tamaño de los organismos. Estos fueron seleccionados aleatoriamente sin ser sustraídos.

Análisis estadísticos: Para detectar cambios en la abundancia de C. abbreviata y C. caribaea a través de todos los meses se utilizó un ANDEVA de medidas repetidas en ambas profundidades con una prueba a posteriori de Bonferroni. La variable densidad fue transformada mediante la raíz cuadrada para normalizar la distribución y homogeneizar la varianza.

Para detectar cambios en el largo de $C$. abbreviata a través de todos los meses se realizó un ANDEVA de medidas repetidas con la prueba a posteriori de Bonferroni. Se utilizó la de T-Student para comparar la abundancia de C. abbreviata a 3 y 6 metros de profundidad.

Se utilizó un ANDEVA de una vía con una prueba a posteriori de Tukey para comparar el tamaño de los individuos de C. abbreviata asociados a las distintas especies coralinas. Se utilizó una prueba de T-Student para comparar los promedios del largo de C. abbreviata y 
C. caribaea asociados a la especie coralina Acropora palmata.

\section{RESULTADOS}

Densidad de $C$. abbreviata y $C$. caribaea: Las densidades de C. abbreviata presentaron ciertas fluctuaciones a lo largo del año en ambos estratos profundidad destacando el mínimo registrado en septiembre a $3 \mathrm{~m}$ de profundidad y enero a $6 \mathrm{~m}$ de profundidad y el máximo obtenido en mayo en ambas profundidades, este último mes fue el único que presentó diferencias significativas (ANDEVA de medidas repetidas, $\mathrm{p}<0.05$ ), y fue con los meses de enero y marzo en la profundidad de $6 \mathrm{~m}$ (Fig. 1).

Se observaron mayores fluctuaciones en las densidades de la transecta somera durante el año, la cual fluctuó entre $1.78 \mathrm{ind} / \mathrm{m}^{2}$ (septiembre) y $6.26 \mathrm{ind} / \mathrm{m}^{2}$ (mayo), sin embargo ninguno de sus valores presentaron diferencias significativas (ANDEVA de medidas repetidas, $\mathrm{p}<0.05$ ), por otro lado la transecta profunda presentó menores fluctuaciones, las cuales estuvieron comprendidas entre $4.23 \mathrm{ind} / \mathrm{m}^{2}$ (enero) y $7.66 \mathrm{ind} / \mathrm{m}^{2}$ (mayo) (Fig. 2). Es importante señalar que mayo fue el mes en el

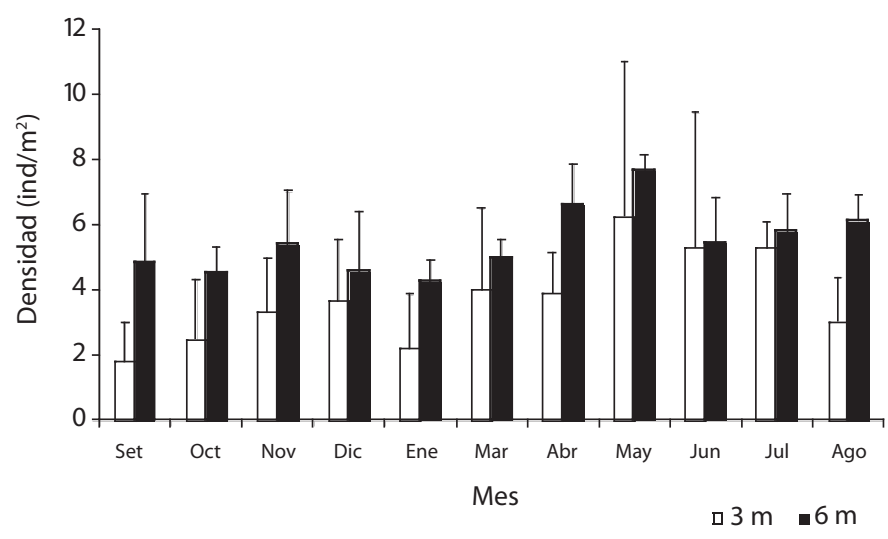

Fig. 1. Densidades de C. abbreviata a lo largo de todo el año a $3 \mathrm{~m}$ (A) y $6 \mathrm{~m}$ (B) de profundidad.

Fig. 1. Densities of C. abbreviata through the year, $3 \mathrm{~m}(\mathrm{~A})$ and $6 \mathrm{~m}$ (B) deep.

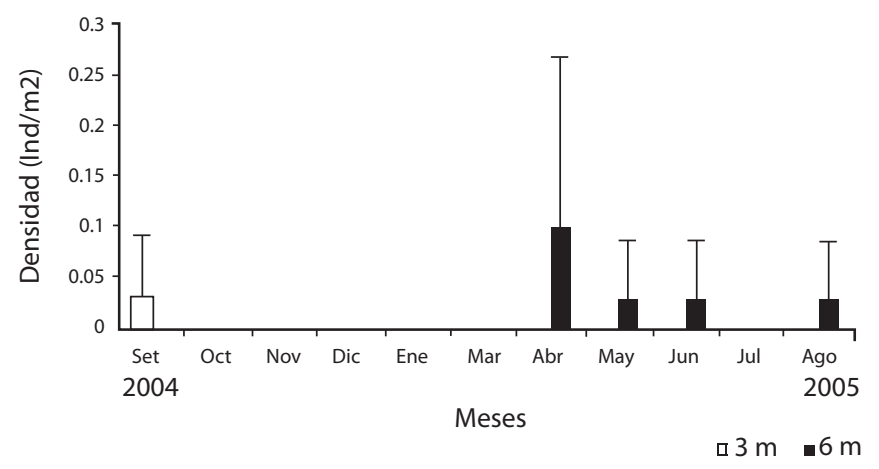

Fig. 2. Densidades de $C$. caribaea $\left(\mathrm{Ind} / \mathrm{m}^{2}\right)$ a lo largo de todo el año a $3 \mathrm{~m}$ y $6 \mathrm{~m}$ de profundidad.

Fig 2. Densities of C. caribaea $\left(\mathrm{Ind} / \mathrm{m}^{2}\right)$ throughout the year, $3 \mathrm{~m}$ and $6 \mathrm{~m}$ deep. 


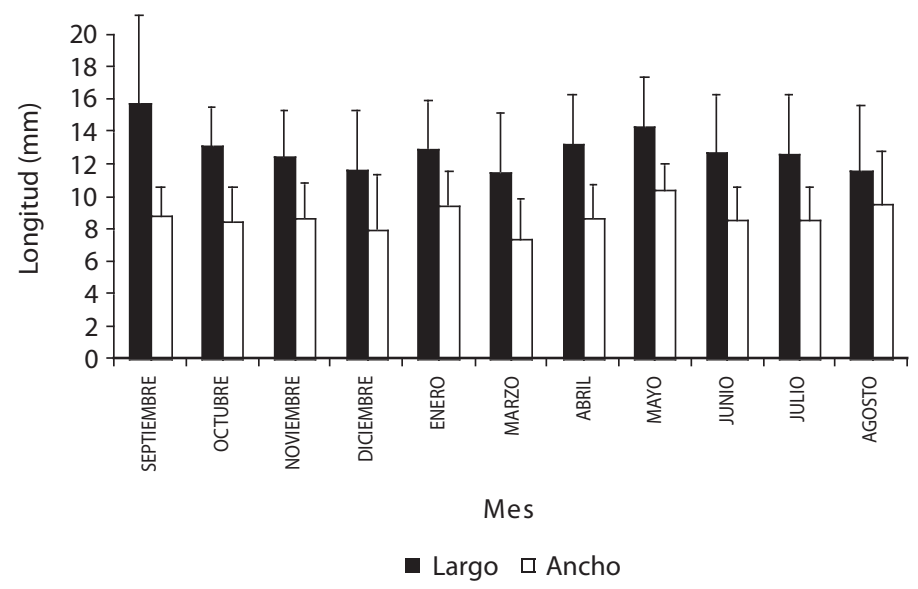

Fig 3. Promedio de ancho y largo de los individuos de C. abbreviata medidos a lo largo del año.

Fig 3. Mean breadth and length of C. abbreviata individuals throughout the year.

cual se registraron las mayores densidades a ambas profundidades. Otro aspecto de relevancia es que las densidades presentadas en la transecta de $6 \mathrm{~m}$ fueron significativamente mayores a las densidades registradas en la transecta de 3 $\mathrm{m}$ (T-Student, $\mathrm{p}<0.05$ ).

Con respecto a la variabilidad temporal de las densidades de $C$. caribaea, en el estrato de 3 $\mathrm{m}$ solo se encontraron individuos durante septiembre de 2004 con una densidad muy baja de $0.03 \mathrm{Ind} / \mathrm{m}^{2}$ y en el estrato de $6 \mathrm{~m}$ solo se registraron individuos en abril a agosto (con excepción de julio) con densidades que oscilaron entre $0.03 \mathrm{Ind} / \mathrm{m}^{2}$ (mayo, junio y agosto) y $0.1 \mathrm{Ind} / \mathrm{m}^{2}$ (abril) (Fig. 2). Sin embargo desde una perspectiva estadística no hubo diferencias importantes en el cambio de las densidades de $C$. caribaea a través del tiempo en ambos estratos de profundidad (ANDEVA medidas repetidas, $\mathrm{p}=0.423$ en cada estrato), como tampoco hubo diferencias entre los promedios totales de las densidades de C. caribaea comparando ambas profundidades (T-Student, $\mathrm{p}=0.158$ ). Esto ocurrió debido a que las densidades de este depredador se mantuvieron muy bajas a través de todo el año evaluado, en ambas profundidades.

Estructura de tallas de $C$. abbreviata y $C$. caribaea: Se observó homogeneidad en los promedios de la longitud de todos los individuos de $C$. abbreviata medidos en todos los meses en cada una de las transectas (Fig. 3), por lo que no registraron diferencias significativas (ANDEVA de medidas repetidas, $\mathrm{p}<0.05$ ), no obstante cuando se discriminó el número de organismos de cada talla se observó que la mayoría de los organismos medidos en todos los meses midieron entre 12 y $16 \mathrm{~mm}$, encontrándose muy pocos individuos por encima de los $20 \mathrm{~mm}$ y por debajo de los $5 \mathrm{~mm}$ (Fig. 4).

Diciembre, marzo y agosto fueron los meses en que se midió la mayor proporción de individuos menores a $9 \mathrm{~mm}(26 \%, 31 \%$ y $38 \%$ respectivamente), y los meses en que se midió la menor proporción fueron mayo y octubre ( $0 \%$ y $8,33 \%$ respectivamente) (Fig. 4). Por otra parte los meses donde se obtuvo una mayor proporción de individuos entre 10 y $15 \mathrm{~mm}$ fueron octubre y noviembre $(83.33 \%$ y $80 \%$ respectivamente) aunque en todos los meses más de la mitad de los organismos estuvieron dentro de este intervalo exceptuando agosto, mes en el cual solo se midió una proporción de $47.6 \%$. En lo que respecta a los individuos por encima de los $15 \mathrm{~mm}$, mayo y julio fueron los meses con mayor proporción de individuos dentro de ese intervalo con $33.33 \%$ y $24 \%$ respectivamente; por otra parte los meses que registraron las menores proporciones dentro de 

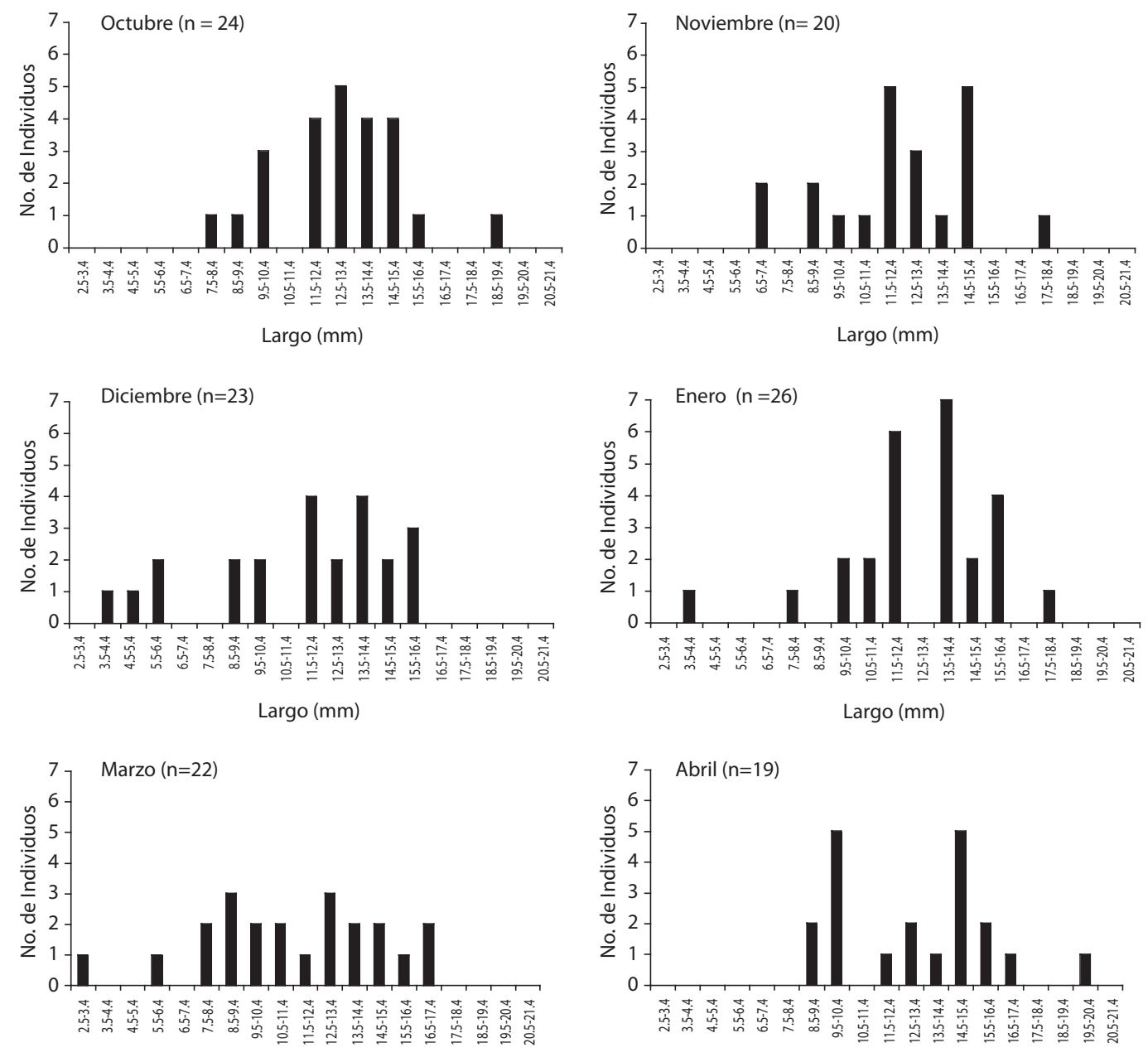

Largo $(\mathrm{mm})$

Largo $(\mathrm{mm})$

Fig 4. Estructura de talla poblacional de $C$. abbreviata a lo largo de un ciclo anual. Solo se midieron ejemplares $(\mathrm{n}=225)$ hallados sobre M. annularis.

Fig 4. Size structure of C. abbreviata throughout the year. We only measured individuals found on M. annularis $(\mathrm{n}=225)$.

dicho intervalo fueron noviembre y octubre con $5 \%$ y $8,33 \%$ respectivamente (Fig. 4 ).

Es importante señalar que además se midieron otros organismos de C. abbreviata asociados a otras especies coralinas en Cayo Sombrero, los cuales no fueron incluidos en la graficación de la estructura de tallas para evitar la variabilidad en el tamaño de los individuos debido a posibles diferencias en la calidad nutricional de las especies coralinas, dicho individuos fueron en total 225 , los cuales se encontraban depredando 6 especies coralinas diferentes, 112 de ellos se encontraban sobre M. annularis, 34 sobre $C$. natans, 40 sobre A. agaricites, 16 sobre $A$. tenuifolia, 23 sobre D. strigosa y 6 sobre $A$. palmata. La longitud promedio de los individuos de C. abbreviata asociados a $C$. natans y D. strigosa fue 

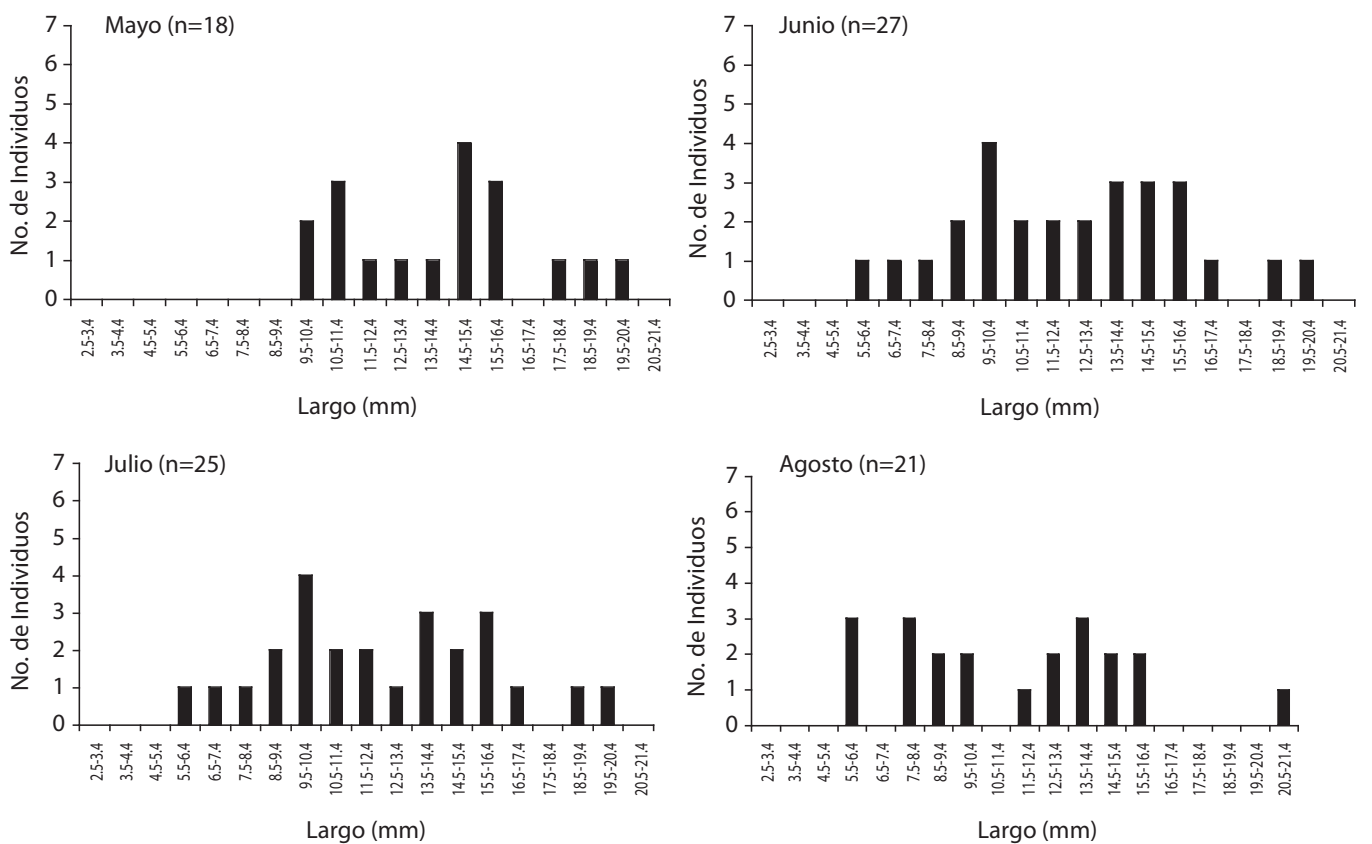

Fig 4. (Continuación) Estructura de talla poblacional de C. abbreviata a lo largo de un ciclo anual. Solo se midieron ejemplares hallados sobre $M$. annularis $(\mathrm{n}=225)$.

Fig 4. (Continued) Size structure of C. abbreviata throughout the year. We only measured individuals found on M. annularis $(n=225)$.

significativamente mayor (ANDEVA de una vía, $\mathrm{p}<0.05)$ a la de los individuos asociados a las especies coralinas M. annularis, A. tenuifolia y A. agaricites. El individuo más pequeño presentó una longitud de $2 \mathrm{~mm}$ y fue encontrado sobre una colonia $A$. agaricites y el más grande fue de $42 \mathrm{~mm}$ fue encontrado sobre una colonia de $C$. natans.

Por otra parte los individuos asociados a M. annularis presentaron una longitud significativamente mayor a los individuos asociados a A. tenuifolia y $A$. agaricites (ANDEVA de una vía, $\mathrm{p}<0.05)$ (Fig. 5). Es importante señalar que los individuos de C. abbreviata encontrados sobre $A$. palmata fueron de mayor longitud a los hallados sobre A. tenuifolia y A. agaricites, sin embargo debido al bajo número de individuos encontrados sobre $A$. palmata las diferencias no fueron estadísticamente significativas (ANDEVA de una vía, $\mathrm{p}<0.05$ ).
Con respecto a la estructura de tallas de C. caribaea, no se realizó una evaluación mensual a lo largo del año debido al bajo número de individuos encontrados, por lo que se midió una muestra de 53 individuos durante abril, estos fueron recolectados sobre la especie $A$. palmata y en su mayoría midieron entre 10 y $15 \mathrm{~mm}$ de largo (62.26\%). No se recolectaron individuos con tallas inferiores a los $6 \mathrm{~mm}$ ni superiores a los $17 \mathrm{~mm}$ (Fig. 6). La talla promedio fue de $11,16 \pm 3,04 \mathrm{~mm}$ con una proporción de $30.19 \%$ de individuos por debajo de los $9 \mathrm{~mm}$ de largo y $7.54 \%$ por encima de $15 \mathrm{~mm}$.

La longitud promedio de C. caribaea fue menor al de C. abbreviata (ambos depredadores tomados de $A$. palmata), dicho resultado no fue significativo (T-Student, $\mathrm{p}<0.05$ ) posiblemente debido al bajo número de individuos de $C$. abbreviata medidos (6), lo que probablemente 


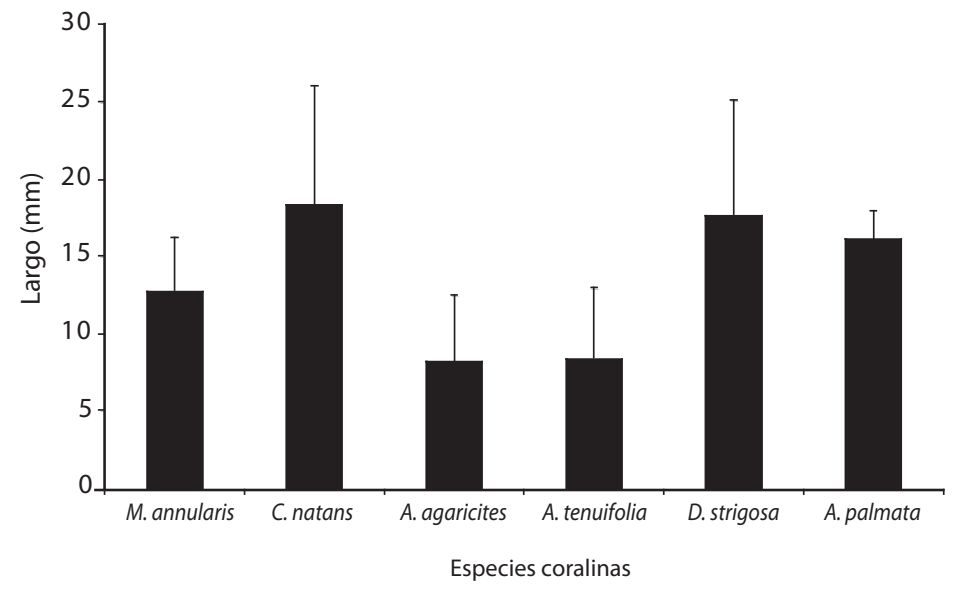

Fig. 5. Longitud de los individuos de C. abbreviata asociados a distintas especies coralinas.

Fig. 5. Lenght size of C. abbreviata individuals associated with various coral species.

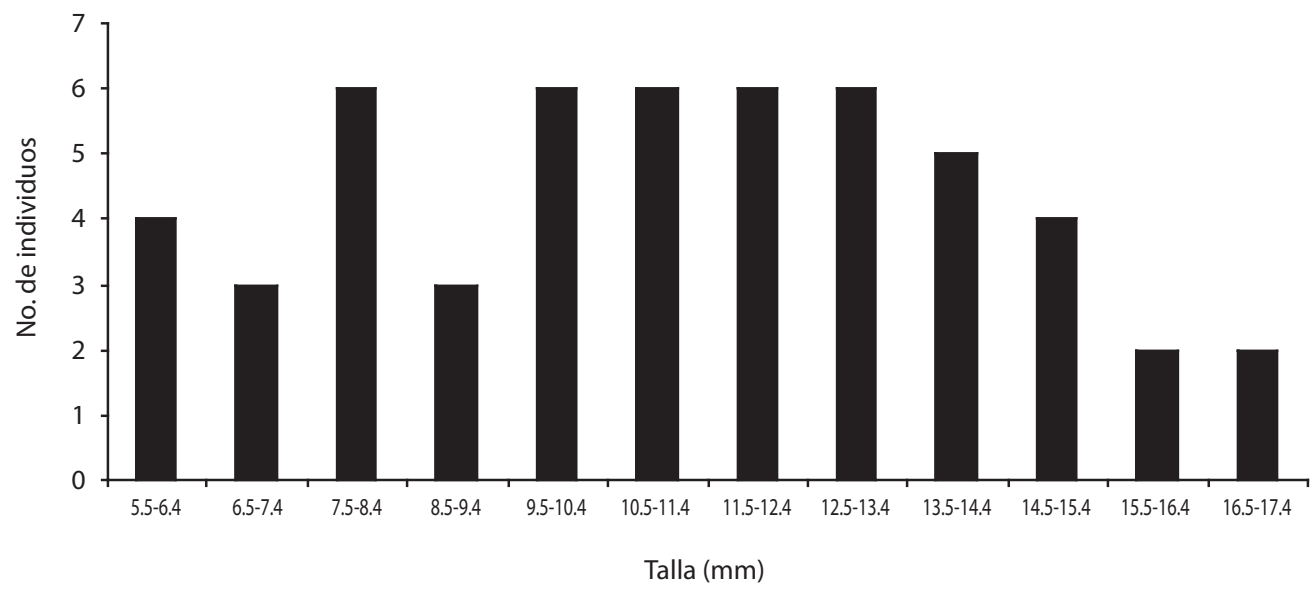

Fig. 6. Estructura de tallas de $C$. caribaea $(\mathrm{n}=53)$ basada en individuos observados durante abril.

Fig. 6. Size structure of C. caribaea individuals $(\mathrm{n}=53)$ measured during April.

generó un incremento en el error aleatorio afectando el análisis estadístico (Fig. 7).

\section{DISCUSION}

La variabilidad temporal en las densidades poblacionales de C. abbreviata pudo obedecer a diversos factores. Turner (1994a) señala que las poblaciones de muchos invertebrados marinos fluctúan considerablemente en su tamaño en intervalos regulares y explica que la variabilidad en el reclutamiento de larvas del molusco coralívoro Drupella cornus, los procesos de establecimiento y la mortalidad temprana durante la etapa post-establecimiento., ya que los adultos son relativamente sedentarios y su migración es poco probable, además indica que estas fluctuaciones pueden surgir debido al 


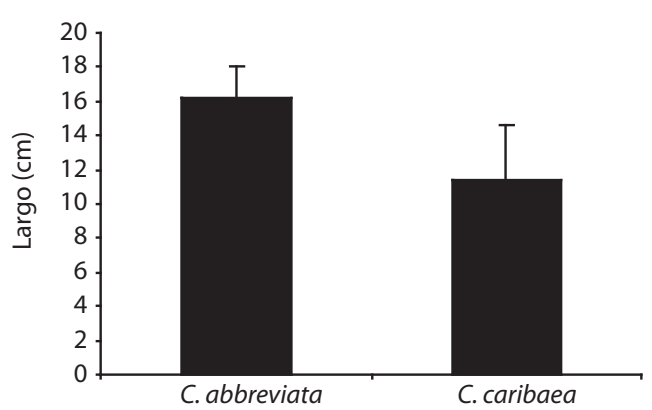

Fig. 7. Longitud de los individuos de C. abbreviata y $C$. caribaea asociados a la especie A. palmata.

Fig. 7. Length size of $C$. abbreviata and C. caribaea individuals associated with $A$. palmata.

diferencial en la probabilidad de supervivencia de cada estadio del ciclo de vida. Igualmente Zann et al. (1987) y Doherty y Davidson (1988) determinaron que el incremento abundancia de Acanthaster planci pudo haber sido causada por el aumento en el reclutamiento de las larvas. Por otra parte Lasker y Coffroth (1988) señalan que los cambios en las densidades de depredadores en el tiempo y espacio pueden ser debido a fluctuaciones espacio-temporales en la disponibilidad, calidad y abundancia de presas octocoralinas, sin embargo en este trabajo no se realizó ninguna evaluación en relación a los cambios en la cobertura coralina a través del tiempo por lo que no se puede descartar esta posibilidad.

Con respecto a las alteraciones de la calidad de las presas, esto podría explicar las razones por las cuales se observaron mayores fluctuaciones en las densidades del estrato de $3 \mathrm{~m}$ en comparación con las del estrato de $6 \mathrm{~m}$, ya que las colonias del estrato somero están sometidas a un mayor impacto turístico (daños mecánicos por anclas, mayor impacto por bañistas) y una mayor influencia de la lluvia por las escorrentías desde el Cayo lo que se puede traducir en disminuciones en la calidad del alimento obligando a C. abbreviata a migrar a mayores profundidades.

Muchas de las aseveraciones señaladas anteriormente podrían explicar las fluctuaciones exhibidas en la población de C. abbreviata de Cayo Sombrero, ya que, al igual que $D$. cornus, este molusco presenta poca movilidad, lo cual hace suponer que la migración de estos individuos es improbable, indicando que la variabilidad en las tasas de reclutamiento y establecimiento podrían ser factores muy importantes que definen la variabilidad temporal de las densidades y dinámica poblacional de estos depredadores. Sin embargo esta condición no se ve reflejada en los promedios totales de la estructura de talla analizada durante todo el año, ya que no se observaron fluctuaciones, sino mas bien una marcada homogeneidad en sus valores promedio por lo que no se registraron diferencias significativas $(\mathrm{p}<0.05)$ entre las tallas de los individuos medidos a través de todo el año.

$\mathrm{Si}$ interpretamos las diferentes clases de tallas registradas de $C$. abbreviata en este trabajo durante todo el año como clases de estadios, entonces los meses con mayor proporción de jóvenes fueron diciembre, marzo y agosto, observándose una elevada proporción de adultos los meses posteriores a estos (enero, abril y septiembre) y reflejando un posible crecimiento de los jóvenes que reclutaron durante los meses anteriores; por otra parte si se considera que la larva se mantienen por un tiempo de 4 a 6 semanas en la columna de agua (según Turner (1994b) las larvas de D. cornus podrían mantenerse durante este tiempo) entonces los mayores eventos de liberación de las larvas pudieron haber sido uno o quizás dos meses antes de dichas temporadas. Es importante señalar que se observó una gran actividad reproductiva de C. abbreviata durante abril, mayo, junio y noviembre, épocas en que se observó en el laboratorio la eclosión de larvas velígera de esta especie, además en reiteradas ocasiones cuando se sustraía un individuo in situ, se observó liberando las puestas en el tejido coralino o en sustrato rocoso, por lo que pudieran haber muchas épocas reproductivas de esta especie en el año lo que pudo haber causado la similitud en los promedios de las densidades y las tallas (en el caso de C. abbreviata) de ambos depredadores durante todo el año evaluado. 
Ward (1965), Wells y Lalli (1977) y Ott (1971, tomado de Wells y Lalli 1977) registran la escasez de jóvenes de C. abbreviata en sus estudios, sugiriendo Wells y Lalli (1977) que estos podrían estar en diferentes hábitats que los adultos o que las poblaciones de estos moluscos se mantienen en una relativa estabilidad con una baja tasa de reclutamiento. Forde (1992), Turner (1994a) y Zann et al. (1987) encontraron que existía un cambio de hábitat entre los jóvenes y los adultos de D. cornus y A. planci.

Durante este trabajo los individuos de C. abbreviata o C. caribaea fueron siempre observados sobre colonias coralinas vivas, a pesar de que se revisaron todos los sustratos, incluyendo escombros coralinos, arena, roca entre otros; esta situación también fue señalada por Baums et al. (2003a) los cuales tampoco pudieron observar a $C$. abbreviata en algún otro punto del arrecife que no fuera sobre tejido coralino vivo.

Turner (1994a) considera que los individuos de $D$. cornus, por debajo de $20 \mathrm{~mm}$ de largo son jóvenes y por debajo de $10 \mathrm{~mm}$ son reclutas con menos de un año de edad y señala que estos últimos son crípticos y se ubican en las bases del coral muerto, donde se protegen de los depredadores y de las corrientes marinas. Forde (1992) explica que los adultos de $D$. cornus, los cuales miden entre 28 y $40 \mathrm{~mm}$ de largo, son comúnmente encontrados en corales "tabulados" del género Acropora o Montipora, por otra parte los reclutas, cuya longitud es de 2 a $22 \mathrm{~mm}$, son encontrados casi exclusivamente sobre especies "digitate" de Acropora; por último señala que la talla intermedia, denominados como jóvenes, son crípticos, siendo encontrados bajo los escombros coralinos.

Otra razón que pudo haber evitado la observación de reclutas en el presente estudio es la selectividad del sustrato por parte de la larva al instante de reclutar, ya que la larva podría seleccionar otras especies de coral que le ofrezcan una mayor heterogeneidad espacial que la proteja de los depredadores.

Es importante señalar que en mayo y junio, meses en que se registraron las mayores densidades de C. abbreviata en ambas profundidades, se observó un gran número de individuos con puestas, que posteriormente en el laboratorio eclosionaron, esta condición podría sugerir que C. abbreviata incrementa sus densidades en épocas reproductivas para aparearse.

Con respecto al tamaño de C. abbreviata y su especie coralina asociada, Baums et al. (2003a) registran individuos de C. abbreviata cuyo largo estuvo comprendido entre $5.8 \mathrm{~mm}$ y $53.6 \mathrm{~mm}$, depredando la especie coralina $A$. palmata estos fueron significativamente más largos que aquellos que fueron recolectados depredando M. annularis. El intervalo de longitudes registrado por Baums et al. (2003b) fue similar al del presente estudio, no obstante, dichos autores encontraron individuos de hasta $53.6 \mathrm{~mm}$ mientras que en el presente estudio el mayor individuo fue de $42 \mathrm{~mm}$. Por otra parte, a diferencia del estudio de Baums et al. (2003a), en el presente trabajo si se encontraron algunos individuos por debajo de los $5 \mathrm{~mm}$, sin embargo fueron observados principalmente sobre $A$. agaricites, A. tenuifolia y un solo individuo sobre $M$. annularis.

Wells y Lalli (1972) registraron la estructura de tallas de individuos de C. abbreviata y $C$. caribaea en Barbados, presentando $C$. abbreviata un intervalo entre los 8.7 y 23.6 $\mathrm{mm}$ de largo con un promedio de $13.5 \pm 1.5$ $\mathrm{mm}$ los machos y $17.6 \pm 1.7 \mathrm{~mm}$ las hembras, y C. caribaea un intervalo de 5.1 y 35.4 $\mathrm{mm}$ con un promedio de $21.5 \pm 2.7 \mathrm{~mm}$ las hembras y $15.2 \pm 2.5 \mathrm{~mm}$ los machos; dichos autores señalan que el largo de las hembras fue significativamente mayor al de los machos en ambas especies y que el promedio de largo de los individuos de C. caribaea de ambos sexos fue mayor que el de $C$. abbreviata, esta última aseveración no coincide con los resultados del presente estudio, ya que, se registró una mayor longitud de C. abbreviata en comparación con C. caribaea; la causa de esta disimilitud en los resultados pudo haber sido debido a que los individuos recolectados de C. abbreviata y C. caribaea por Wells y Lalli (1972) fueron tomados de especies coralinas diferentes, ya que $C$. abbreviata fue recolectada de la especie 
coralina $M$. annularis y los de C. caribaea fueron tomados sobre Gorgonia sp lo que pudo haber generado las diferencias de tamaño debido a las diferencias nutricionales.

Baums et al. (2003b) señalan que existen diferencias interespecíficas en la calidad del alimento de los corales, dicha calidad se encuentra asociada con la cantidad de carbono y nitrógeno del tejido coralino, cantidad y potencia de los nematocitos y estructura esqueletal (Glynn y Krupp 1986, Gochfeld, 2004), tales diferencias nutricionales deben traducirse en el tamaño que puede alcanzar un individuo adulto de Coralliophila. Bruckner et al. (1997) registraron que los individuos de C. abbreviata recolectados sobre $A$. palmata fueron más largos que aquellos individuos recolectados sobre otras especies coralinas como $M$. annularis, $D$. strigosa, C. natans y D. labyrinthiformis.

La talla mínima de madurez reproductiva y la época de reproducción de C. abbreviata no está clara (Wells y Lalli 1977), sin embargo existe evidencia de que se reproduce en Barbados durante ciertas épocas del año, Ward (1965) registró cápsulas de huevos en agosto, aunque no en diciembre; Ott (1971, tomado de Wells y Lalli 1977) registró cápsulas entre febrero y julio; y Wells y Lalli (1977) encontraron en julio cápsulas de huevos en estadio de velígera, sin embargo también observaron cápsulas con larvas aún no maduras para eclosionar en ese mes. Con respecto a $D$. cornus Turner (1994a) indica que el mayor evento de reclutamiento lo detectó durante enero y febrero de 1991.

Todos los meses evaluados (exceptuando mayo, en el caso de C. abbreviata) reflejan que la abundancia de $C$. abbreviata y $C$. caribaea no varía ampliamente a través del año, lo cual podría indicar que su efecto depredador sobre el arrecife de Cayo Sombrero es constante en relación a sus densidades y que no posee épocas reproductivas claras que podrían generar incrementos abruptos en sus densidades.

\section{RESUMEN}

C. abbreviata y C. caribaea son moluscos coralívoros del Mar Caribe. Evaluamos la variabilidad temporal de la abundancia y los cambios en la estructura de talla de $C$. abbreviata y $C$. caribaea en Cayo Sombrero, Venezuela (septiembre 2004-agosto 2005). Se colocaron transectas fijas de 50 metros de longitud a profundidades de tres y seis metros cada una, paralelas a la costa. Se evaluó mensualmente durante un año la densidad de ambos depredadores y se seleccionaron 30 individuos de C. abbreviata para medirlos cada mes. Las densidades de $C$. abbreviata en la transecta somera fluctuaron entre $1.78 \mathrm{ind} / \mathrm{m}^{2}$ en septiembre y $6.26 \mathrm{ind} / \mathrm{m}^{2}$ en mayo; las densidades de C. abbreviata en la transecta profunda estuvieron comprendidas entre $4.23 \mathrm{ind} / \mathrm{m}^{2}$ en enero y $7.66 \mathrm{ind} / \mathrm{m}^{2}$ en mayo. Las densidades de $C$. caribaea fluctuaron en la transecta somera entre $0 \mathrm{Ind} / \mathrm{m}^{2}$ (todos los meses menos septiembre) y 0.03 $\mathrm{Ind} / \mathrm{m}^{2}$ (septiembre) mientras que la transecta profunda fluctuó entre $0 \mathrm{Ind} / \mathrm{m}^{2}$ (octubre a marzo y julio) y $0.1 \mathrm{Ind} /$ $\mathrm{m}^{2}$ (abril). La mayoría de los individuos de C. abbreviata midieron entre 12 y $16 \mathrm{~mm}$ de longitud. El largo de la mayoría de los individuos de C. caribaea estuvo comprendido entre 10 y $15 \mathrm{~mm}$.

Palabras clave: Coralliophila sp, coralívoros, variabilidad temporal, abundancia, estructura de talla, Parque Nacional Morrocoy.

\section{REFERENCIAS}

Baums, I., M. Miller \& A. Szmant. 2003a. Ecology of a corallivorous gastropod, Coralliophila abbreviata on two scleractinian hosts I: Population structure of snails and corals. Mar. Biol. 142: 1083-1092.

Baums, I.., M. Miller \& A. Szmant. 2003b. Ecology of a corallivorous gastropod Coralliophila abbreviata on two scleractinian hosts II: feeding, respiration and growth. Mar. Biol. 142:1093-1102.

Bone, D., D. Pérez, A. Villamizar, P. Penchaszadeh \& E. Klein. 1998. Parque Nacional Morrocoy, Venezuela. In B. Kjerfve (Eds.). CARICOMP - Caribbean Coral Reef, Seagrass and Mangrove sites. UNESCO, Paris, 151-159.

Bruckner, R., A. Bruckner \& E. Williams. 1997. Life history strategies of. Coralliophila abbreviata Lamarck (Gastropoda: Coralliophilidae) on the southwest coast of Puerto Rico. Proceedings of the Eigth International Coral Reef Symposium, Panamá. 1: 627-632.

Doherty, P. \& J. Davidson. 1988. Monitoring the distribution and abundance of juvenile Acanthaster planci in the central Great Barrier Reef, p.131-136. In Proceedings of the Sixth International Coral Reef Symposium (eds.). Townsville, Australia.

Forde, M. 1992. Populations, behavior and effects of Drupella cornus on the Ningaloo Reef, Western 
Australia, p. 45-50. In S. Turner (eds.). Drupella cornus: a synopsis. Western Australia Department of Conservation and Land Management, CALM Ocassional Paper No. 3/92.

Glynn, P. \& D. Krupp. 1986. Feeding biology of a Hawaiian sea star corallivore, Culcita novaeguineae Muller \& Troschel. J. Exp. Mar. Biol. Ecol. 96: 75-96.

Gochfeld, D. 2004. Predation-induced morphological and behavioral defenses in a hard coral: implications for foraging behavior of coral-feeding butterflyfishes. Mar. Ecol. Prog. Ser. 267: 145-158.

Hayes, J. 1990. Prey preference in a Caribbean corallivore, Coralliophila abbreviata (Lamarck) (Gastropoda, Coralliphilidae). Bull. Mar. Sci. 47: 557-560.

Lasker, H., M. Coffroth and L. Fitzgerald. 1988. Foraging patterns of Cyphoma gibbosum on octocorals: the roles of host choice and feeding preference. Biol. Bull. (Woods Hole). 174: 254-266.

Lewis, J. 1960. The coral reefs and coral communities of Barbados. Can. J. Zool. 38: 1133-1145.

Miller, A. 1981. Cnidarian prey of the snails Coralliophila abbreviata and C. caribaea (Gastropoda: Muricidae) in Discovery Bay, Jamaica. Bull. Mar. Sci. 31: 932-934.

Miller, M. 2001. Corallivorous snail removal: evaluation of impact on Acropora palmata. Coral Reefs 19: 293-295.
Miller, M., I. Baums, D. Williams \& A. Szmant. 2002. Status of candidate coral Acropora palmata, and its snail predator in the upper Florida Keys National Marine Sanctuary: 1998-2001. NOAA Technical Memorandum NMFS-SEFSC-479.

Ott, B. 1971. Hermatypic coral predation at Barbados, West Indians, by Coralliophila abbreviata (Gastropoda, Prosobranchia) and Hermodice carunculata (Polychaeta, Errantia). Unpublished M. Sc. Thesis, McGill University, Montreal, Canadá.

Turner, S. 1994a. The biology and population outbreaks of the corallivorous gastropod Drupella on Indo-Pacific reefs. Oceanogr Mar. Biol. Annu. Rev.32: 461-530.

Turner, S. 1994b. Spatial variability in the abundance of the corallivorous gastropod Drupella cornus. Coral Reefs. 13: 41-48.

Ward, J. 1965. The digestive tract and its relations to feeding habitats in the stenoglossan prosobranch Coralliophila abbreviata (Lamarck). Can. J. Zool. 43: 447-464.

Wells, F. \& C. Lalli. 1977. Reproduction and brood protection in the Caribbean gastropods Coralliophila abbreviata and C. Caribaea. J. Moll. Stud. 43: 79-87.

Zann, L., J. Brodie, C. Berryman \& M. Naqasima. 1987. Recruitment, ecology, growth and behavior of juvenile Acanthaster planci (L.) (Echinodermata: Asteroidea). Bull. Mar. Sci. 41: 561-575. 